\title{
Development of Contact Type Positioning-free Supplier and Charger
}

\author{
Daisuke Tanaka ${ }^{a}$, Masaharu Moritsugu ${ }^{a}$, Shun Inoue ${ }^{a}$, Mayu Yamanaka ${ }^{a}$, Lifeng Zhang ${ }^{\text {a }}$ \\ ${ }^{a}$ Kyushu Institute of Technology, 1-1 Sensui-cho Tobata-ku Kitakyushu-shi Fukuoka-ken, 804-8550, Japan \\ *d.tanaka@boss.ecs.kyutech.ac.jp
}

\begin{abstract}
In near future, many autonomous robots will be used to perform, to help with housework and to take care of the elderly people. However, their power consumption is very high, so it is significant to supply the power to robots efficiently. The three major power supply methods have been used; "wired style", "internal rechargeable battery" and "change style". Each method has some advantages and disadvantages. We focused on "change style" but it requires the high-level control to change battery correctly. If we could solve this problem, the robot's efficiency will become better. In this study, we will show that the electrical current controller using diode-bridge can flow the electrical current only one direction without depending position and the automatic battery charging system can use only putting the battery on it and charge the battery automatically.
\end{abstract}

Keywords: robot, charge, positioning-free

\section{Introduction}

In the near future, many autonomous robots will be used to perform, to help with housework and to take care of the elderly people. Especially, instead of the helper, they will be action in nursing home for the elderly. Therefore, recently, the number of elderly is increasing rapidly and the amount of such facilities is also increasing accordingly. However, robots' power consumption is so high because of using a lot of motors that supplying the power to robots efficiently is very significant.

The three major power supply methods is the following three types: "wired style", "internal rechargeable battery" and "change style". Each style has some advantages and disadvantages. For example, in wired style, one of the advantages is that robots can use larger amount of electric power than other style and one of the disadvantages is that robots are limited moving area because of power supply cable. In this case, considering management cost and environmental performance, we focused on "change" because many robots are required to work for a long time in the same area. Actually, when the robots' battery needs to be charged, it is essential operation to judge whether battery's electrode position is right or not correctly. Therefore, it is more profitable to control the electric electrical current by simple circuit.

In this study, We clarify that the electrical current control circuit using diode-bridges is not only simple but also able to flow the electrical current to one direction without depending position and the automatic battery charger can charge the battery automatically only putting on it.

\section{The electrical current supplier}

An electrode's pattern and a schematic of the electrical current control circuit using diode-bridge are shown in Fig.1. Fig.1.(a) is for robots and Fig.1.(b) is for the battery. The electrodes for robots in Fig.1.(a) is consists of four square electrode and each square electrode in Fig.1.(a) is connected with same number's terminal in Fig.2. Only putting the battery on electrodes in Fig.1(a) such as in Fig.3, this can control the current flow. The mechanism is that; When a battery having electrode in Fig.1.(b) is put on Fig.1.(a), if electrode is positive, a B side diode in Fig.2. is ON but if negative, an A side diode is ON. As a result, the electrical current flow direction doesn't change. Even if the battery is put on some electrodes at the same time, the direction of electrical current flow is not affected. 


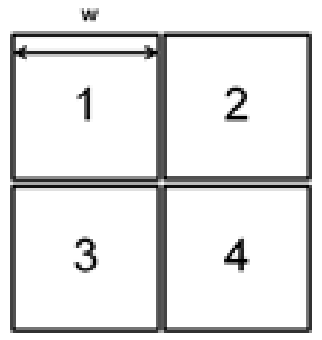

(a) Square electrodes

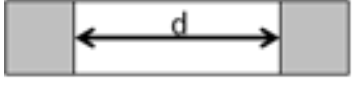

(b) Battery's electrodes
Fig.1. Electrode's style

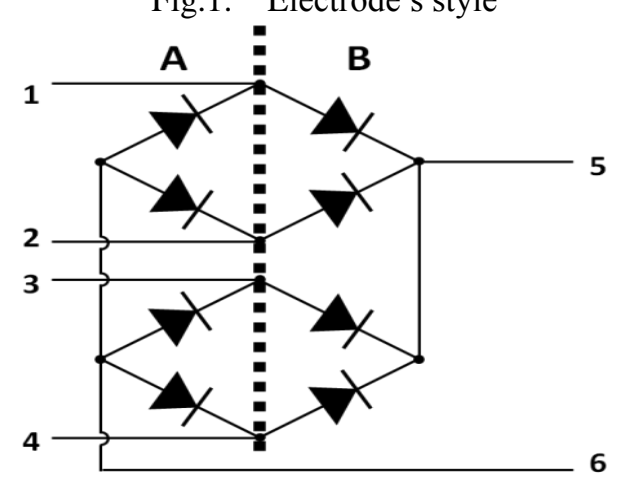

Fig.2. Electrical current control circuit

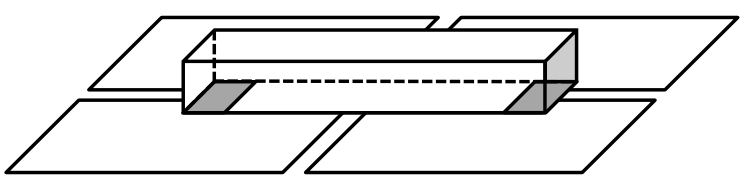

Fig.3. How to use the system

Unfortunately, this system has a problem that when the battery's electrodes are put on the one square electrode at the same time, battery is a short. In addition to that, the battery's position is limited. To achieve above mechanism and to prevent a short, it is necessary to satisfy the following inequality.

$$
\mathrm{d}>\sqrt{2} \mathrm{w} \cong 1.5 \mathrm{w}
$$

If the circuit satisfies this inequality, a short never happen. Furthermore, if the size of the square electrode is much shorter than the distance between battery's electrodes, we can use this system for every type battery.

\section{Positioning-free automatic charger}

Positioning-free Automatic charger is more convenient than previous battery charger. Many kinds of automatic battery charging systems are already developed and sold from many companies but all of them have mechanical parts such as motor, gear, relay and so on. Compared with normal type charger, the probability of breakdown is so high due to mechanical parts that it is important to decrease the probability. To achieve it, we developed the automatic battery charging system. The main characteristics of this system are as follows;

- The circuit is simple and the scale is small.

- We can use it only putting the battery on.

- This can charge without depending position.

- Various type batteries can use it.

We used PIC (Peripheral Interface Controller), analog multiplexers, photo-coupler and FETs. A block diagram is shown in Fig.4. and a schematic is shown in Fig.4. Multiplexers' ports are connected with not only each other but also same number square electrode in Fig.1.(a).

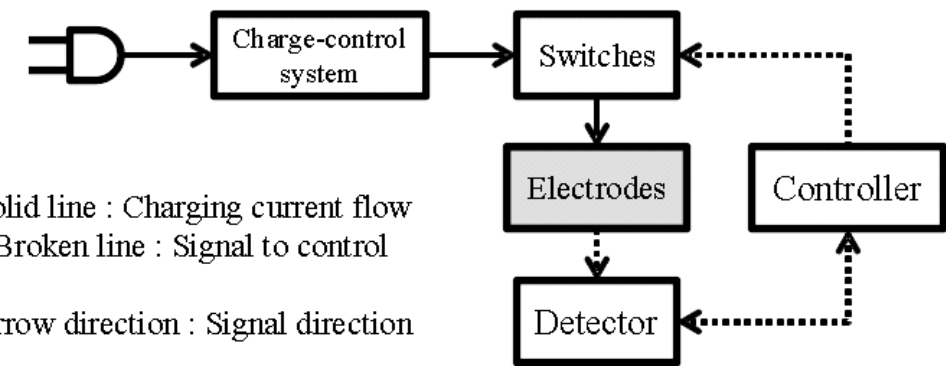

Fig.3. A flow chart of detection system.

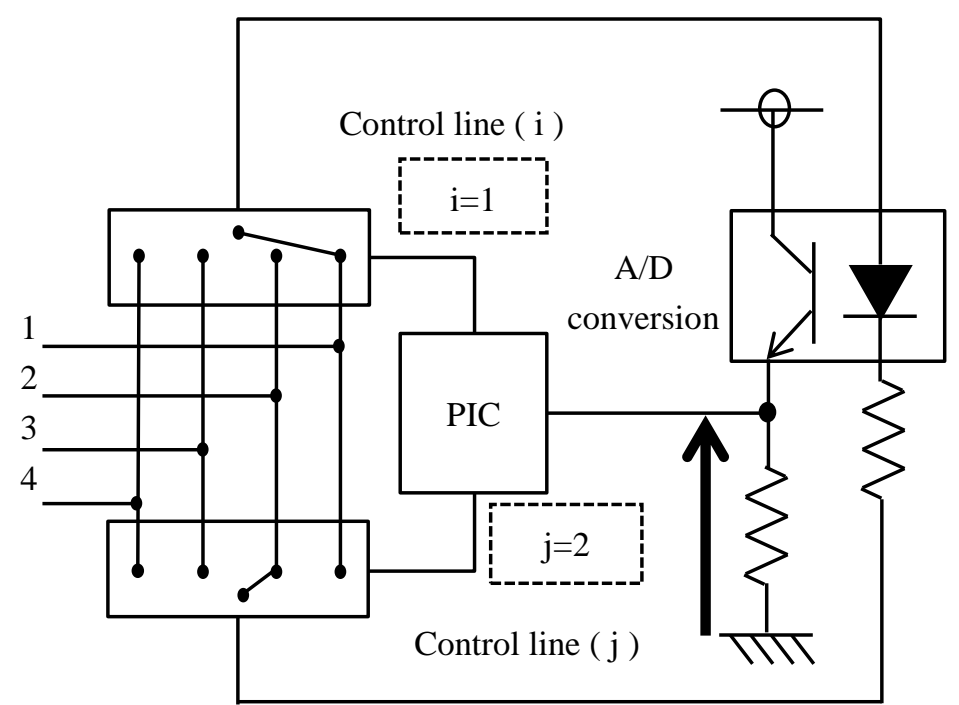

Fig.4. A schematic of the detection system. 


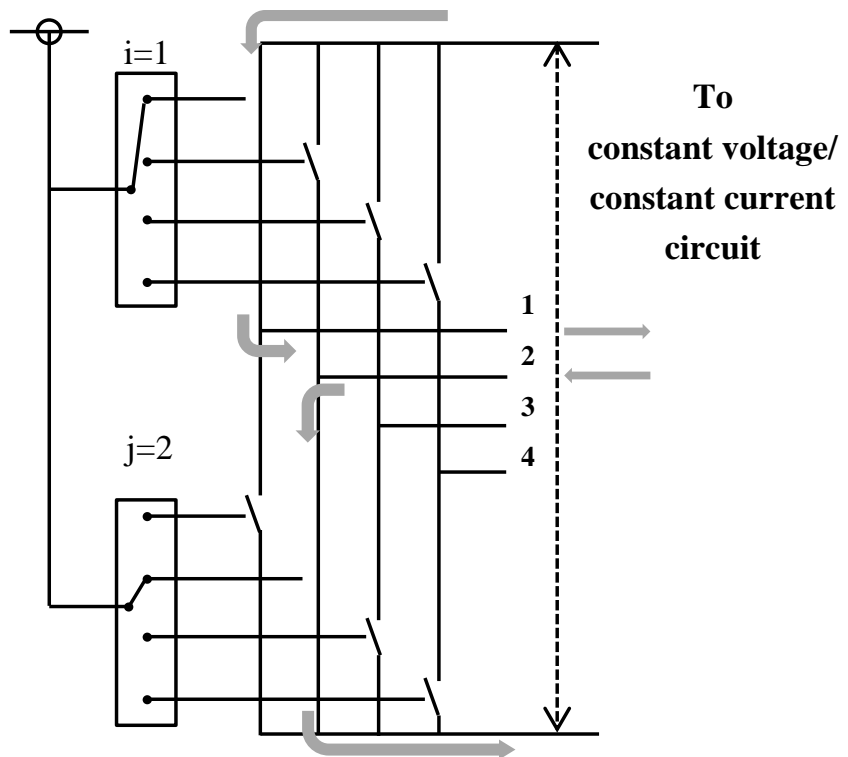

Fig.5. A schematic of the switch system.

The control processes are as follows;

(1) PIC decides the value of variable $\mathrm{i}$ and $\mathrm{j}$. In my processes, the variable $i$ never equals the variable $j$ to prevent a short.

(2) As shown in Fig.4, PIC sends them to multiplexers and both common terminals of multiplexers are connected with photo-couplers.

(3) If the battery is put on the electrode, the electrical current flows, LED is lit. Therefore, photo-transistor turns on, the voltage difference between ground and emitter of photo-transistor is converted by A/D conversion terminal.

(4) If the result of $\mathrm{A} / \mathrm{D}$ conversion is zero, the LED isn't lit. It means that the current doesn't flow, the direction is opposite or the battery isn't put on the electrode. Conversely, if the result isn't zero, the LED is lit. It means that the current flows and PIC can recognize the battery's polarity.

(5) After PIC recognized the battery's polarity, as shown in Fig.5, the multiplexers are used not as the multiplexers but as the decoders to control switches.

(6) If the switch turns on, battery charging starts. In the beginning, the battery is charged by constant current charging. As the battery's voltage level approaches approximately $4 \mathrm{~V}$, the charging method is changed from constant current charging to constant voltage charging.

Recently, Li-ion rechargeable battery is very common and is used for many things such as mobile phone, PC and so on. However, when Li-ion battery is charged, the charger has to control current and voltage more careful than other type batteries because Overcharge may happen the breakage

\section{Simulation and experimental result}

\subsection{The electrical current control circuit}

To confirm my suggestion, we used the circuit as shown in Fig.6 and experimented.

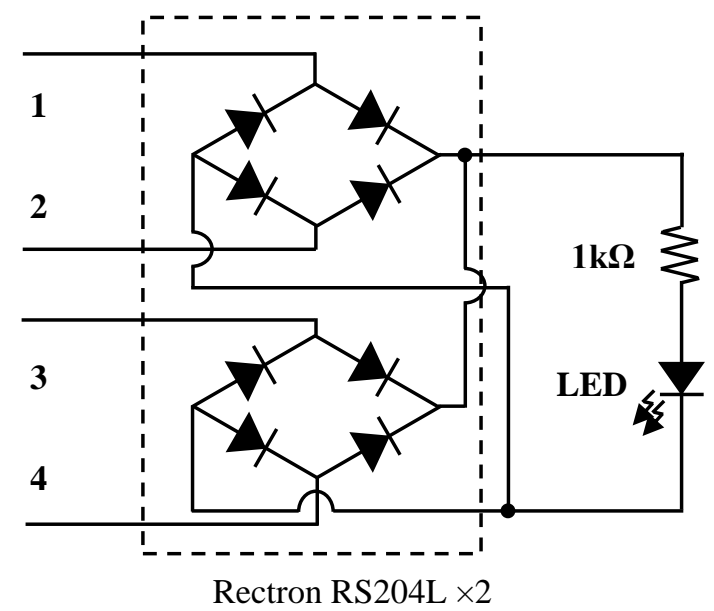

Fig.6. A schematic for experiment

The patterns of square electrodes are 16, the patterns of battery electrodes are 2 and the total patterns are 32(16*2). We confirmed whether the LED was lit or not in all patterns. If the direction of electrical current is not unidirectional, it means that the current direction depends on battery's position.

As a result of experimentation, the LED was lit in all patterns. In other words, the direction of electrical current is unidirectional and it doesn't depend on position.

\subsection{Automatic battery charging system}

In addition to the circuit of Fig.4, we used 7 segment LEDs and BCD-to-Seven segment decoder IC to check whether my program was working correctly. (7 segment LED and BCD-to-Seven segment decoder IC were used only for checking program errors.)

We made 7 segment LED module and connected it with PIC. In the program, the variable $\mathrm{i}$ and $\mathrm{j}$ are displayed when the result of $\mathrm{A} / \mathrm{D}$ conversion is not zero. For example, if the variable $i$ is $1, j$ is 2 and A/D conversion is not zero, the number " 12 " is displayed on the 7 segment LEDs such 
as Fig.7. Furthermore, the ten's place shows the position of battery's positive electrode and the one's place shows the position of battery's negative electrode. Therefore, by watching the number, we could check whether the program is working correctly. The number is displayed on 7 segment LED module only the value of $\mathrm{V}$ is over the threshold. (The threshold is determined in the PIC's program.)

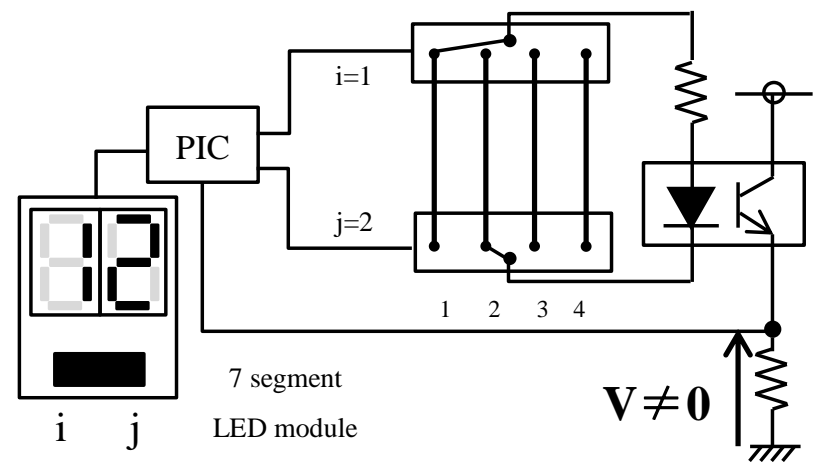

Fig.7. A schematic of 7 segment LED module

\section{Conclusion}

From the experimental result, proposed circuit could flow electric current without depending battery's electrode position and could detect the battery's electrode polarity automatically. At the moment, the electrical current control circuit and the automatic battery charging system could develop but constant current / constant voltage circuit couldn't so I'm going to develop it, combine them and invent the whole system.

\section{References}

(1) A.Kawamura, K.Ishida, and J.Hirai : "Wireless Transmission of Power and Information Through One High-Frequency Resonant AC Link Inverter for Robot Manipulator Applications", IEEE Trans.Industrial Applicat., Vol.32, No.3, pp.503-508 (1996)

(2) C.S. Wang, O.H. Stielau and G.A. Covic : "Design Considerations for a Contactless Electrical Vehicle Battery Charger", IEEE Trans.Industrial Electronics, Vol.52, No.5, pp.1308-1314(2005)

(3) van Wageningen, Dries, and Toine Staring. : "The Qi wireless power standard." Power Electronics and Motion Control Conference (EPE/PEMC), 2010 14th International. IEEE, pp. S15-25 (2010)

(4) Qi, Q., and C. Chakrabarti. : "Improving the battery performance of ad-hoc routing protocols." Signal
Processing Systems Design and Implementation, 2005. IEEE Workshop on. IEEE, pp. 720-725. (2005)

(5) Boscaino, Valeria, et al. "A wireless battery charger architecture for consumer electronics." Consumer Electronics-Berlin (ICCE-Berlin), 2012 IEEE International Conference on.pp.84-88 (2012)

(6) Jian, Xiao Zhi, and Han Zhen Yu. "A novel wireless charging system for movable telephone with printed-circuit-board windings of different structure and shape respectively". In: Electrical Machines and Systems, 2007. ICEMS. International Conference on. IEEE, pp.1283-1285. (2007) 